Article

\title{
Long term data on obstructive sleep apnea treatment in insom- nia and anxiety related disorders - importance in the post- covid19 era?
}

\author{
Silvia Gavliakova ${ }^{1,2}$, Vladimira Bellova ${ }^{2}$, Diana Vitazkova ${ }^{2}$, Peter Bullo ${ }^{2}$
}

How to cite this paper: Gavliakova,

S.; Bellova, V.; Vitazkova, D.; Bullo,

P. Long term data on obstructive

sleep apnea treatment in insomnia and anxiety related disorders - importance in the post-covid19 era?

Open Journal of Medical Sciences 2021, 1(1), 1-9.

https://doi.org/10.31586/ojms.2021.01 0101

Received: May 01, 2021

Accepted: June 06, 2021

Published: June 07, 2021

Copyright: (c) 2021 by the authors. Submitted for possible open access publication under the terms and conditions of the Creative Commons Attribution (CC BY) license (http://creativecommons.org/licenses /by/4.0/).

\author{
${ }^{1}$ Jessenius Faculty of Medicine in Martin, Comenius University in Bratislava, Martin, Slovakia \\ ${ }^{2}$ Clinic of Pneumology and Phthisiology, University Hospital Martin and Jessenius Faculty of Medicine CU, \\ Martin, Slovakia \\ *Correspondence: silvia.gavliakova@gmail.com; Tel.: 00421944476497
}

\begin{abstract}
Patients with comorbid neurotic and anxiety disorders are more receptive of the discomfort accompanying continuous positive airway pressure therapy than average obstructive sleep apnea patients. The purpose of the study was to analyze short-term and long-term continuous positive airway pressure therapy adherence data of patients with obstructive sleep apnea and comorbid anxiety and stress-related dyssomnias, as this group of disorders is expected to rise in the post-covid era. Study retrospectively analyzed clinical outcomes of obstructive sleep apnea patients. All subjects with obstructive sleep apnea were diagnosed based on in-lab video polysomnography, further referred to CPAP titration and were invited for regular follow-up visits. The results showed that subjects with comorbid obstructive sleep apnea and anxiety-related disorders used ventilation therapy more hours per day (6,690 hours/day vs. 5,000 hours/day, ${ }^{* * * *} \mathrm{p}<0,0001$, anxiety ( $\left.\mathrm{n}=19\right)$ vs. controls $(n=60))$. Patients from the anxiety group remained longer in our therapy program $(7,086$ years vs. 2,905 years, $\left.{ }^{* * * *} \mathrm{p}<0,0001\right)$ and had markedly better control over their weight, as the body-mass index of the control group increased by $+1,065 \mathrm{~kg} / \mathrm{m} 2$ per year and the body-mass index in the examination group increased only by $+0,296 \mathrm{~kg} / \mathrm{m} 2$ every year. Our data document that obstructive sleep apnea patients on ventilation therapy, who do not have enough control over their increasing body weight might highly benefit from therapy approaches similar to patients with anxiety-related dyssomnias and that management of obstructive sleep apnea is not an obstacle in concomitant treatment of neurotic and anxiety-related dyssomnias.
\end{abstract}

Keywords: obstructive sleep apnea, obesity, anxiety, dyssomnia, insomnia

\section{Introduction}

Recent months of covid-19 pandemic have undisputable impact not only on our healthcare and economic systems, but also, the pandemic impacts prominently our mental health. Frontline workers are dealing with depression, anxiety and stress in their workplace and in their homes. The mental health burden of home confinement and imminent concern about social and financial safety impacts unfavorably the lives of communities in pandemic-struck countries. Among all these mentioned facts, we assume a relatively considerable cluster of patients with an increasing ratio of sleep disorders, as the prevalence of dyssomnias during pandemic times and economic crisis typically rises. The very first covid-19-related data are already being published [1, 2]. Other covid-19 specific is the proportion of long-lasting lockdowns and movement restrictions, affecting and changing our lifestyles. People have tendency to become more sedentary as a natural consequence of pandemic restrictions. Sedentary lifestyle than leads to weight gain, unhealthy life-style choices, habits and unhealthy sleeping hygiene $[3,4,5,6]$. The 'obesity epidemic' will therefore be still a growing concern globally and obesity-related respiratory disease (e.g., 
obesity related sleep disturbed breathing) will reflect an increasing proportion of patients in both inpatient and outpatient settings [7]. A part of obstructive sleep apnea (OSA) patients might be falsely diagnosed with insomnia and are sent for OSA screening and consultation at the appropriate time or vice versa, also because dyssomnia and insomnia periods might have seasonal character and patients themselves are not able to identify their problem during the routine consultation with their sleep specialists. Moreover, some authors posit that the frequency of anxiety in obstructive sleep apnea patients is higher than in the general population regardless of the gender, or that it is more likely that obstructive sleep apnea patients present with anxiety and depression than the typical symptoms [8].

As many authors realize the temporary imminence of the post covid19 era, we might shortly see the impacts of covid19-related life-style changes such as local lockdowns and movement-restriction on our health. As a natural consequence, we might encounter higher incidence of obstructive sleep apnea risk factors - obesity and/or benzodiazepine sleep aids overuse, etc. Current scientific data, while still scarce, advocate an integrated and multidisciplinary approach that seems superior over the isolated treatment of each sleep disorder alone $[9,10]$.

\subsection{Purpose of the study}

Our main pool of interest were obstructive sleep apnea patients in specific groups of comorbid sleep disorders: insomnias not due to a substance or known physiological condition, primary and adjustment insomnias, psychophysiological insomnia, insomnias and sleep disorders in anxiety, panic or somatoform disorders - (further referred to as IAP "insomnias, anxiety, panic disorders").

The key purpose of the study was to evaluate short-term and long-term continuous positive airway pressure (CPAP) therapy adherence data of patients with obstructive sleep apnea and comorbid dyssomnias. Specifically, we were interested in the outcomes of a cluster of patients with comorbid obstructive sleep apnea and dyssomnias related to difficulties in initiating and/or maintaining sleep (e.g., idiopathic insomnia, anxiety syndrome, panic disorder etc.). The important goal of the designed project was to analyze available long-term retrospective data with focus on the most actual global problems. The outcomes will help us in the fight against "post-covid syndromes" in the new era (such as obesity related obstructive sleep apnea syndrome, or dyssomnias in relation to internalizing disorders), so we can be better prepared to predict, address and manage arising sleep problems of our patients. Taking all these into account, our hypothesis was that patients with obstructive sleep apnea (OSA) and comorbid insomnias and internalizing disorders will experience more difficulties with ventilation therapy adherence over the course of time in comparison to a control group of patients without any known psychiatric disorders.

Excluded from the study were patients diagnosed with hypersomnia episodes in major depressive disorders, bipolar disorders, schizoaffective disorders, narcolepsy, other hypersomnia and chronic fatigue syndrome.

\section{Materials and Methods}

First consultation regarding obstructive sleep apnea screening was in a form of meeting and a dialogue, to put focus on OSA differential diagnosis, Epworth sleepiness scale assessment and introducing the patients into healthy sleep hygiene techniques. If obstructive sleep apnea was suspected, patients were admitted to in-lab video polysomnography and followingly CPAP titration.

Dyssomnias and anxiety syndromes were diagnosed prior to obstructive sleep apnea and the IAP patients were instructed to continue with their routine pharmacological therapy also on the day of polysomnography. Effect of CPAP therapy was tried if the apnea/hypopnea index was over 15 events per hour of sleep. CPAP was considered effective, if the patient used the device at least $70 \%$ of the time, more than 4 hours per day and the 
residual apnea/hypopnea index on CPAP was under 10 respiratory events per hour. Subjects who started CPAP were invited for regular outpatients visits every 6-12 months to monitor their condition and therapy adherence. Patients were divided into two groups and matched for AHI, BMI, Epworth score and age at the time of obstructive sleep apnea diagnosis and CPAP initialization.

Statistical analysis and data visualizations were performed using GraphPad Prism. Data are represented as medians, if not stated otherwise. Unpaired nonparametric MannWhitney test was used to compare two sets of data. Probability analysis and Kaplan-Meier curves were used to portray best CPAP compliance probability and the risk of gaining weight for the duration of CPAP therapy program (hazard ratio, log-rank comparison). To inspect relationships between data, Spearman $\mathrm{r}$ correlation coefficient was calculated and results were considered significant if two-tailed $p$ value was $<0,05$ (confidence interval 95\%). The research and manipulation with medical records data was conducted according to the principles of the Declaration of Helsinki. The study was designed as retrospective research on data available hence the authors did not seek ethical committee approval or informed consent. All data analyzed were collected as part of routine diagnosis and treatment. Patients were diagnosed and treated according to national guidelines. The tests and recordings mentioned in this article are essential for confirming diagnosis, classifying and treating patients. It is done for each patient as part of routine care.

\section{Results}

\subsection{Examination group - IAP}

The database collected over the past 10 years was composed of 315 medical examination reports of 60 IAP patients (14 female, 46 male) who complied with our inclusion criteria for the test group. Continuous positive airway therapy was initiated in 36 patients with apnea/hypopnea index over 15 events/hour Table 1, but only 19 out of 36 [52,78\%] subjects with comorbid obstructive sleep apnea and IAP were able to continue with the therapy in the long term. Most commonly reported complaints, negatively affecting patient's adherence to the CPAP therapy, were feelings of anxiety about the mask interface disturbing their sleep and feelings of claustrophobia. Some of the patients did not tolerate even low CPAP pressure settings of $4 \mathrm{cmH} 2 \mathrm{O}$. For detailed analysis of CPAP adherence, we have collected 189 medical examination reports from 19 patients with comorbid obstructive sleep apnea and IAP ( 3 female, 16 male) and compared the data with 60 apnea/hypopnea index matched subjects in the control group.

Table 1. Apnea/hypopnea index in patients with problems with maintaining and/or initiating sleep, based on video polysomnography results.

\begin{tabular}{|c|c|c|}
\hline $\begin{array}{c}\text { Comorbid obstructive sleep apnea } \\
\text { syndrome (OSA) }\end{array}$ & $\begin{array}{c}\text { AHI } \\
\text { (events/hour } \\
\text { s }\end{array}$ & $\mathrm{n}=60$ \\
\hline No OSA & $0,00-4,99$ & $14[23,33 \%]$ \\
\hline Mild OSA & $5,00-14,99$ & $10[16,67 \%]$ \\
\hline Moderate OSA & $15,00-29,99$ & $17[28,33 \%]$ \\
\hline Severe OSA & $\geq 30,00$ & $19[31,67 \%]$ \\
\hline \multicolumn{2}{|c}{} \\
\hline
\end{tabular}

\subsection{Control group}

Our database consisted of 5039 medical records regarding OSA and CPAP from 1003 patients (205 female and 798 male), which were recorded from subjects who visited our sleep laboratory over the past 10 years. Out of this database, we have selected a control group consisting of data from 60 apnea/hypopnea index matched patients with no known psychiatric disorder (14 female, 46 male, 315 medical examination reports regarding OSA 
and CPAP), AHI=33,90 (24,05-68,14; 25\%-75\%) vs. AHI=38,83 (23,73-70,22; 25\%-75\%); $\mathrm{p}=0,9501$; controls versus IAP.

\subsection{Short-term data}

Patients in both groups were invited for a first appointment within 6-12 months after the initialization of CPAP and later continued in regular visits every 6-12 months. Analysis of the most recent data (first 8-16 months of CPAP therapy) showed that within the first months of continuous positive airway pressure therapy, there was no significant difference in deltaBMI (body mass index at the time of obstructive sleep apnea diagnosis compared to actual body mass index after the initialization of CPAP) between the two groups (deltaBMI $+3,096$ vs. $+2,045 ; p=0,4625$, controls vs. IAP). Subjects in both of the studied groups had a tendency to gain some amount of weight. Similarly, there was no significant difference in CPAP daily compliance in the first 2 years of CPAP $(5,000(2,973-$ $7,130 ; 25 \%-75 \%)$ hours/day vs. 4,368 (2,624-5,966; $25 \%-75 \%)$ hours/day; IAP vs. controls, $\mathrm{p}=0,1383)$.

\subsection{Long-term data}

Long-term data showed an interesting trend. Despite the fact, that after the initial CPAP trial only $52,78 \%$ of the IAP patients with comorbid obstructive sleep apnea and IAP agreed to continue in CPAP treatment, over the period of time, they complied significantly better with the treatment in term of daily hours and also, they tended to remain longer in the therapy program.

Patients with comorbid obstructive sleep apnea and IAP used their CPAP more hours per day (6,690 hours/day vs. 5,000 hours/day, ${ }^{* * * *} p<0,0001$ IAP vs. controls), Figure 1. Analysis of compliance criteria (CPAP daily use over 4,000 hours per day) showed no significant difference in the ratio of compliant versus non-compliant patients $(\mathrm{p}=0,7473)$ between the groups. However, patients from the IAP group remained more years in our therapy program $\left(7,086\right.$ years vs. 2,905 years, $\left.{ }^{* * * *} p<0,0001\right)$.

Median of daily CPAP use (MED \pm IQR)

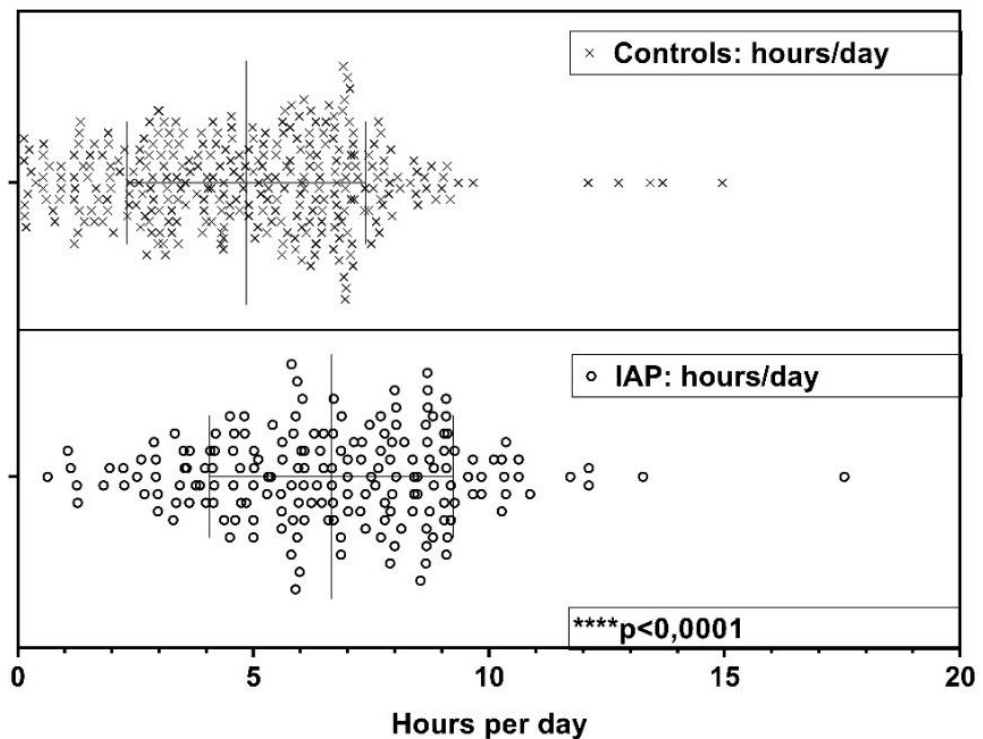

Figure 1. Daily CPAP usage, long-term. Graphical representation of continuous positive airway pressure therapy usage by patients with sleep apnea displayed in average hours per day. Information was derived from long-term data, as determined in regular medical examination reports from every 12 months. Patients diagnosed with problems with initiating and/or maintaining sleep (IAP) prior to obstructive sleep apnea diagnosis used CPAP ventilator notably more hours per day. Although patients in both groups could be categorized as compliant (median of daily use was over 4.0 hours per day for patients in both groups). 
Initial body-mass indexes of both groups at the time of obstructive sleep apnea diagnosis were similar (BMI=31,14 kg/m2 (27,71-37,59; 25\%-75\%) vs. BMI=31,06 kg/m2 (24.21$37,58 ; 25 \%-75 \%), p=0,5639$, controls vs. IAP) and their body weight increased over time. But interestingly, patients in the IAP group had markedly better control over their weight. In total, body weight of patients in the IAP group increased as the median of deltaBMI was $+2,045 \mathrm{~kg} / \mathrm{m} 2(-1,734-4,665 ; 25 \%-75 \%)$. DeltaBMI median in the control group was $+3,096 \mathrm{~kg} / \mathrm{m} 2(-2,210-6,253 ; 25 \%-75 \%),{ }^{* * * *} \mathrm{p}<0,0001$. Meaning, that the body-mass index of the control group increased by $+1,065 \mathrm{~kg} / \mathrm{m} 2$ per year and the body-mass index in the IAP group increased only by $+0,296 \mathrm{~kg} / \mathrm{m} 2$ every year, while subjects in both examined groups reached sufficient CPAP daily hours.

There was no significant difference in age between our subjects. Median age of IAP group at the time of sleep apnea diagnosis was 56,00 years $(47,00-60,00 ; 25 \%-75 \%)$ and median age of the control group was 55,50 years $(47,25-62,00 ; 25 \%-75 \%), p=0,5031$.

Probability analysis and Kaplan-Meier curves in Figure 2 support hypothesis, that patients in IAP group have higher probability to meet compliance criteria (CPAP $\geq 4$ hours/day) over time compared to the control group (log-rank, $\left.{ }^{* * * *} \mathrm{p}<0,0001\right)$. On the other hand, the probability to gain weight seems to be significantly higher in the control group (log-rank, ${ }^{* * * *} p<0,0001$, Figure 3).

Compliance probability $\geq 4$ hours per day

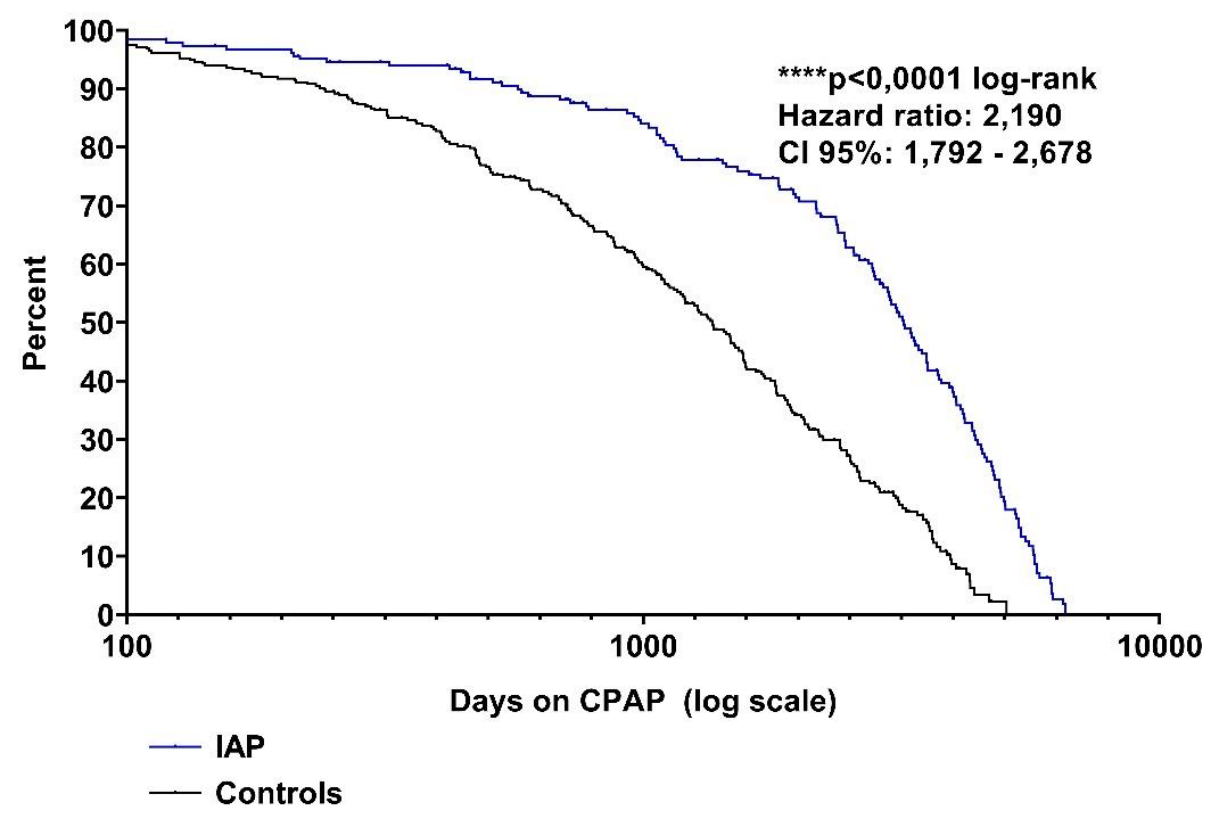

Figure 2. Probability of sufficient CPAP daily compliance. CPAP compliance is commonly referred to as sufficient if the patient is able to use the CPAP on average of 4 hours/day or more. KaplanMeier estimate shows the probability of good compliance over the course of time in patients within the IAP group and controls. Patients in the IAP group not only used their device longer daily average hours but also the probability to stay compliant within the course of time was remarkably higher.

\subsection{Polysomnography parameters and subjective sleepiness from Epworth scale}

There was no significant difference in the baseline sleep parameters recorded from videopolysomnography prior to OSA diagnosis. All the patients had similar sleep efficiency $-87,42 \%$ in IAP and $87,17 \%$ in the control group, $p=0,9955$. Also, deep sleep efficiency was consistent between the groups ( $21,44 \%$ for IAP, $19,34 \%$ for controls, $\mathrm{p}=0,6449$ ). Further, there was no difference in sleep latency, wake periods after sleep onset, arousal index, or total number of spontaneous and respiratory related arousals etc. Subjectively, 
patients in both groups reported their daily sleepiness as increased, scoring over 10 points on the Epworth scale, $\mathrm{p}>0,999$.

Correlation analysis showed, that the CPAP usage hours of subjects in the control group significantly correlated with the deltaBMI $\left(\mathrm{r}=0,2273 ;{ }^{* * * *} \mathrm{p}<0,0001\right)$. This effect was not present in the IAP patients, as the Spearman correlation coefficient was $r=0,049$ $(\mathrm{p}=0,505)$.

\section{Probability of increase of body-mass index over time}

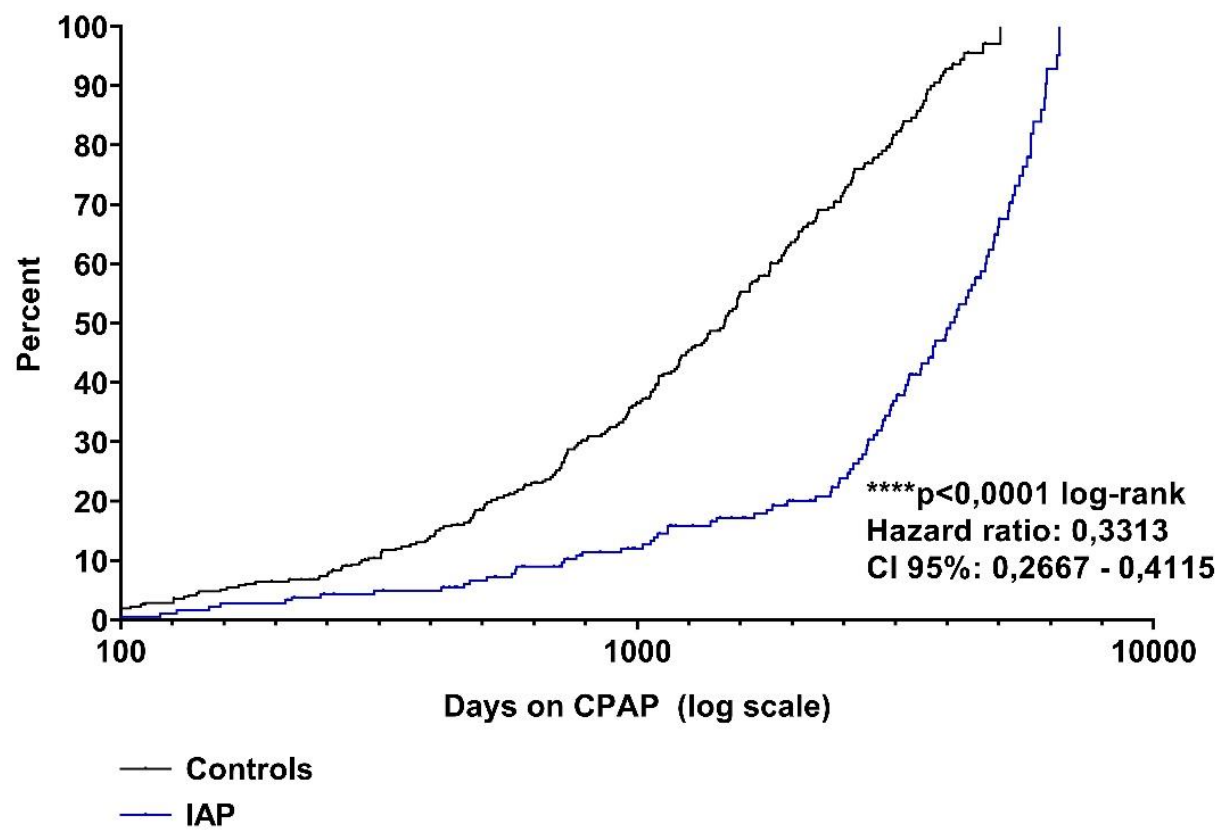

Figure 3. The hazard ratio of increasing body-mass index over time in obese obstructive sleep apnea patients with comorbid obstructive sleep apnea and problems with initiating and/or maintaining sleep seem to have much better control over their body weight. Patients with obstructive sleep apnea and no known psychiatric disorder increased their weight significantly more over the time while continuing CPAP treatment with sufficient daily compliance.

\section{Discussion}

Total prevalence of comorbid IAP syndromes and obstructive sleep apnea is still underrecognized and the coincidence of both groups of disorders is often overlooked [11]. Our results document rather a high proportion of IAP patients [47,22\%], who are discouraged from continuous positive airway pressure therapy since their first in-lab titration night. As the IAP patients on CPAP were highly compliant with the ventilatory treatment in the long-term scale, we are confident that the method of introduction of the patients to their therapy program is one of the most vital variables. Based on these results and based on the newest conveniences of technology, we will decide to take the benefits of telemedicine in the future and to implement home-titration methods via telehealth interfaces. In this way, patients will win more time to familiarize themselves with the CPAP in a home environment. IAP patients in particular could highly profit from telemedicine and hometitration trials and such data will have high medical value also in the future research.

Interestingly, our short-term data from the first 16 months of CPAP in apnea/hypopnea index-, body-mass index-, Epworth score- and age-matched subjects did not reveal any significant differences between the groups regarding daily CPAP usage or weight changes. As we recorded similar polysomnography sleep parameters (such as sleep latency, arousal index etc.) for both groups, we might deduce that the primary therapy of IAP patients was adequate. Logically, average CPAP usage patterns correlate with 
sleeping habits of adherent obstructive sleep apnea patients. Scientific analyses suggest that a greater percentage of patients will achieve normal functioning with longer nightly CPAP durations, but what constitutes adequate use varies between different outcomes [12].

Surprisingly, our long-term results documented that subjects in the IAP cohort achieved significantly higher daily CPAP usage hours with a median of 6,690 hours per day and if the IAP patients agreed to initiate long-term home CPAP therapy, they remained considerably longer in the therapy program in comparison to the controls. We speculate that IAP patients benefited from pharmacological treatment and/or cognitive behavioral therapy strategies targeted at successful management of their primary disease. In the long term, IAP patients could have more experience in overcoming emerging unexpected complications via psychotherapy techniques and those approaches were carried over as well to more effective management of challenging situations related to comorbid obstructive sleep apnea. Such postulate is also supported by the comparison of delta MI parameters between the control and IAP group. We calculated the change of body-mass index over time and the delta demonstrated significantly higher weight gain in the group of patients with no psychiatric diagnosis. Body-mass index of controls increased by $+1,065$ $\mathrm{kg} / \mathrm{m} 2$ per year and the body-mass index in the IAP group increased only by $+0,296 \mathrm{~kg} / \mathrm{m} 2$ every year. Obesity and weight gain together shape a multifactorial phenomenon, which may have different causes, such as incorrect nutritional and dietary habits, psycho-social aspects and sedentary life-style [13]. In our cohort, IAP patients on CPAP seemed to have significantly better control over factors leading to dis-management of obesity risk factors, leading to decreased probability of weight gain over time (Figure 3). Adequate and welltimed treatment of primary diagnosis undisputedly played the most important role for IAP patients. On the other hand, we can suspect that CPAP-adherent obstructive sleep apnea patients, who are not able to manage their weight gain in the long term, might benefit from similar psychotherapy techniques or even pharmacotherapy as patients treated for anxiety-related and internalizing disorders.

Importantly, the authors must mention several limitations which come with the design of this study. The research was conducted as a retrospective, observational and single-centered which could potentially lead to some amount of subject selection and data collection bias. Authors did not intervene with the course of the study and also, they were not able to actively recruit more individuals into the study cohort.

There are published numerous studies focusing on comorbid obstructive sleep apnea, sleep deprivation and anxiety/depression symptoms. Those studies analyzed the bidirectional relationship, symptoms severity, suggest therapy options and agree on urgent need of deeper research in this field $[8,9,10,11]$. Our observational study is first of its kind to evaluate both short-term and more importantly also the long-term clinical outcomes and CPAP ventilation therapy adherence of patients with comorbid obstructive sleep apnea and dyssomnias related to anxiety/internalizing disorders. Furthermore, until today there was no scientific evidence and no published data on long-term CPAP adherence and body-mass index changes in patients with comorbid obstructive sleep apnea and dyssomnias. The authors hypothesize benefits of telemedicine in future CPAP titration studies of patients suffering with anxiety and internalizing disorders, as the telemedicine will bring options for home-based multi-night CPAP titration and potentially more patients can benefit from individualized patient-centered therapy approaches.

\section{Conclusions}

This study was designed to encourage sleep specialists to search out and identify groups of dyssomnia patients with possible comorbid obstructive sleep apnea syndrome who will have a potential to benefit from CPAP therapy. Our data clearly document positive long-term adherence to CPAP therapy also in neurotic and anxiety-related sleep dis- 
orders with comorbid OSA. We further suggest consideration of telemedicine as an appropriate step and solution for CPAP-non-adherent patients with comorbid obstructive sleep apnea and anxiety-related and internalizing disorders. Our data document that OSA patients on CPAP, who do not have enough control over their increasing body weight might highly benefit from therapy approaches similarly to patients with anxiety-related and internalizing disorders.

Author Contributions: Conceptualization, S.G., V.B., D.V. and P.B.; methodology, S.G. and V.B.; validation, S.G., V.B., D.V. and P.B.; formal analysis, S.G.; investigation, S.G.; resources, S.G.; data curation, S.G.; writing - original draft preparation, S.G.; writing-review and editing, S.G.; visualization, S.G.; supervision, V.B.; project administration, S.G. and V.B. All authors have read and agreed to the published version of the manuscript.

Funding: "This research received no external funding"

Data Availability Statement: Data is available on request from the corresponding author.

\section{Acknowledgments: None}

Conflicts of Interest: "The authors declare no conflict of interest." "No funders had any role in the design of the study; in the collection, analyses, or interpretation of data; in the writing of the manuscript, or in the decision to publish the results".

\section{References}

[1] Pappa, S., Ntella, V., Giannakas, T., Giannakoulis, V. G., Papoutsi, E., \& Katsaounou, P. (2020). Prevalence of depression, anxiety, and insomnia among healthcare workers during the COVID-19 pandemic: A systematic review and meta-analysis. Brain, Behavior, and Immunity, 88. https://doi.org/10.1016/j.bbi.2020.05.026

[2] Salari, N., Hosseinian-Far, A., Jalali, R., Vaisi-Raygani, A., Rasoulpoor, S., Mohammadi, M., Rasoulpoor, S., \& Khaledi-Paveh, B. (2020). Prevalence of stress, anxiety, depression among the general population during the COVID-19 pandemic: a systematic review and meta-analysis. Globalization and Health, 16(1). https://doi.org/10.1186/s12992-020-00589-w

[3] Altena, E., Baglioni, C., Espie, C. A., Ellis, J., Gavriloff, D., Holzinger, B., Schlarb, A., Frase, L., Jernelöv, S., \& Riemann, D. (2020). Dealing with sleep problems during home confinement due to the COVID-19 outbreak: Practical recommendations from a task force of the European CBT-I Academy. Journal of Sleep Research, e13052.

[4] Di Renzo, L., Gualtieri, P., Pivari, F., Soldati, L., Attinà, A., Cinelli, G., Leggeri, C., Caparello, G., Barrea, L., Scerbo, F., Esposito, E., \& De Lorenzo, A. (2020). Eating habits and lifestyle changes during COVID-19 lockdown: an Italian survey. Journal of Translational Medicine, 18(1), 229.

[5] Huang, Y., \& Zhao, N. (2020). Generalized anxiety disorder, depressive symptoms and sleep quality during COVID-19 outbreak in China: a web-based cross-sectional survey. Psychiatry Research, 288, 112954.

[6] Stanton, R., To, Q. G., Khalesi, S., Williams, S. L., Alley, S. J., Thwaite, T. L., Fenning, A. S., \& Vandelanotte, C. (2020). Depression, Anxiety and Stress during COVID-19: Associations with Changes in Physical Activity, Sleep, Tobacco and Alcohol Use in Australian Adults. International Journal of Environmental Research and Public Health, 17(11). https://doi.org/10.3390/ijerph17114065

[7] Meurling, I. J., Shea, D. O., \& Garvey, J. F. (2019). Obesity and sleep: a growing concern. Current Opinion in Pulmonary Medicine, 25(6), 602-608.

[8] Rezaeitalab, F., Moharrari, F., Saberi, S., Asadpour, H., \& Rezaeetalab, F. (2014). The correlation of anxiety and depression with obstructive sleep apnea syndrome. Journal of Research in Medical Sciences: The Official Journal of Isfahan University of Medical Sciences, 19(3). https://pubmed.ncbi.nlm.nih.gov/24949026/

[9] Brock, M. S., \& Mysliwiec, V. (2018). Comorbid insomnia and sleep apnea: a prevalent but overlooked disorder. Sleep \& Breathing = Schlaf \& Atmung, 22(1). https://doi.org/10.1007/s11325-018-1628-4

[10] Janssen, H.C.J.P., Venekamp, L. N., Peeters, G. A. M., Pijpers, A., \& Pevernagie, D. A. A. (2019). Management of insomnia in sleep disordered breathing. European Respiratory Review: An Official Journal of the European Respiratory Society, 28(153). https://doi.org/10.1183/16000617.0080-2019

[11] Diaz, S. V., \& Brown, L. K. (2016). Relationships between obstructive sleep apnea and anxiety. Current Opinion in Pulmonary Medicine, 22(6), 563-569.

[12] Weaver, T. E., Maislin, G., Dinges, D. F., Bloxham, T., George, C. F. P., Greenberg, H., Kader, G., Mahowald, M., Younger, J., \& Pack, A. I. (2007). Relationship Between Hours of CPAP Use and Achieving Normal Levels of Sleepiness and Daily Functioning. Sleep, 30(6), 711. 
[13] Marcellini, F., Giuli, C., Papa, R., Tirabassi, G., Faloia, E., Boscaro, M., Polito, A., Ciarapica, D., Zaccaria, M., \& Mocchegiani, E. (2009). Obesity and body mass index (BMI) in relation to life-style and psycho-social aspects. Archives of Gerontology and Geriatrics, 49 Suppl 1. https://doi.org/10.1016/j.archger.2009.09.029 